\title{
Bulimia nervosa em adolescentes do sexo feminino
}

\author{
Bulimia nervosa in female adolescents
}

Bulimia nerviosa en adolescentes

\author{
Juliana Pereira de Almeida \\ ORCID: https://orcid.org/0000-0002-9330-9618 \\ Universidade Nilton Lins, Brasil \\ E-mail: jpalmeida22@hotmail.com \\ Karen Celiane das Chagas Cardoso \\ ORCID: https://orcid.org/0000-0003-0926-4347 \\ Universidade Nilton Lins, Brasil \\ E-mail: karenceliane@gmail.com
}

\begin{abstract}
Resumo
A bulimia nervosa (BN) é uma doença caracterizada por compulsão alimentar descontrolado. O objetivo geral do artigo é destacar, através de uma revisão da literatura, a importância do acompanhamento nutricional na recuperação das adolescentes com Bulimia Nervosa. Aponta-se que objetivos específicos são: elencar como os padrões estéticos afetam o modo de vida das adolescentes que sofrem com Transtornos de Comportamento Alimentares (TCA), conceituar a bulimia nervosa e suas classificações; e destacar o papel da boa alimentação. Para a realização deste trabalho, a metodologia adotada foi levantamento bibliográfico, do tipo revisão narrativa, realizada através de artigos científicos, obtidos por meio das bases de dados: SciELO, Pubmed, Google acadêmico entre outros especialistas que abordam esse assunto. Foram inelegíveis da amostra os artigos publicados e que não estão disponíveis de forma gratuita e não apresentaram o texto na íntegra, artigos que não apresentavam relação direta com o tema, estudos repetidos, além de pesquisas não publicadas entre 2014 e 2021. Conclui-se que os fatores envolvidos na manutenção do transtorno BN são biológicos como predisposição genética, gênero. Os psicológicos incluem impulsividade, perfeccionismo, baixa autoestima, entre outros. A bulimia nervosa implica no tratamento individualizado, porém acompanhado por uma equipe equipes multiprofissionais. Várias ferramentas terapêuticas podem ser utilizadas para o tratamento de pacientes com bulimia nervosa que inclui aconselhamento dietético individualizado que garante um estado nutricional adequado.

Palavras-chave: Bulimia Nervosa; Transtornos Alimentares; Alimentação Saudável.
\end{abstract}

\begin{abstract}
Bulimia nervosa $(\mathrm{BN})$ is a disease characterized by uncontrolled binge eating. The general objective of the article is to highlight, through a literature review, the importance of nutritional follow-up in the recovery of adolescents with Bulimia Nervosa. It is pointed out that specific objectives are to list how aesthetic patterns affect the way of life of adolescents suffering from Eating Behavior Disorders (ACT), conceptualize bulimia nervosa and its classifications; and highlight the role of good nutrition. To carry out this work, the methodology adopted was a bibliographic survey, of the narrative review type, carried out through scientific articles, obtained through the databases: SciELO, Pubmed, Google academic among other experts who address this subject. The articles published and that are not available free of charge and did not present the full text, articles that were not directly related to the theme, repeated studies, and unpublished research between 2014 and 2021 were ineligible. It is concluded that the factors involved in the maintenance of BN disorder are biological as a genetic predisposition, Gender. Psychological scans include impulsivity, perfectionism, low self-esteem, among others. Bulimia nervosa implies individualized treatment but accompanied by a multidisciplinary team. Several therapeutic tools can be used for the treatment of patients with bulimia nervosa which includes individualized dietary counseling that ensures an adequate nutritional status.
\end{abstract}

Keywords: Bulimia Nervosa; Eating Disorders; Healthy Eating.

\section{Resumen}

La bulimia nerviosa (BN) es una enfermedad caracterizada por atracones incontrolados. El objetivo general del artículo es resaltar, a través de una revisión de la literatura, la importancia del seguimiento nutricional en la recuperación de adolescentes con Bulimia Nerviosa. Se señala que los objetivos específicos son: enumerar cómo los patrones estéticos afectan la forma de vida de los adolescentes que padecen Trastornos de la Conducta Alimentaria (TCA), conceptualizando la bulimia nerviosa y sus clasificaciones; y destacar el papel de una buena nutrición. Para la realización de este trabajo, la metodología adoptada fue una encuesta bibliográfica, del tipo revisión narrativa, realizada a través de artículos científicos, obtenidos a través de las siguientes bases de datos: SciELO, Pubmed, académico de Google y otros especialistas que abordan este tema. La muestra no fue elegible para artículos publicados que no están disponibles de 
forma gratuita y no presentaban el texto completo, artículos que no tenían una relación directa con el tema, estudios repetidos e investigaciones inéditas entre 2014 y 2021. del trastorno de BN son biológicos, como predisposición genética, género. Los psicológicos incluyen impulsividad, perfeccionismo, baja autoestima, entre otros. La bulimia nerviosa implica un tratamiento individualizado, pero acompañado de un equipo de equipos multidisciplinares. Se pueden utilizar varias herramientas terapéuticas para tratar a los pacientes con bulimia nerviosa que incluyen un asesoramiento dietético individualizado que asegura un estado nutricional adecuado.

Palabras clave: Bulimia Nerviosa; Trastornos de la Alimentación; Alimentación Saludable.

\section{Introdução}

A bulimia nervosa (BN) é uma doença caracterizada por compulsão alimentar descontrolado, essas ações, por sua vez, apresentam comportamentos compensatórios que visam manter o peso corporal e a aparência desejados, usar laxantes, vômitos, dietas e exercícios físicos exagerada (Ferreira, 2021). A BN é considerada um Transtornos de Comportamento Alimentares (TCA) e sua prevalência entre jovens do sexo feminino e de 1 a 1,5\%, atingindo seu pico no fim da adolescência e início da idade adulta, a prevalência e maior entre adultos (Sebastião, Sampaio \& Barbosa, 2018).

Os adolescentes são afetados porque estão em uma fase da vida que estão passando por mudanças físicas, ocasionadas por alterações hormonais e o desenvolvimento do corpo. Entretanto, estas mudanças estão sendo fortemente influenciados pelas tendências sociais e culturais, que promovem a busca pelo corpo ideal. Porém essa busca pelo está fazendo esses jovens desenvolverem TCA ocasionado pela insatisfação com seu físico (Ludewig et al., 2017).

Como visto, na atualidade o padrão estético de beleza corpóreo é diferente do propagado antigamente. Há uma supervalorização de um corpo magro, definido e musculoso como sinal de saúde e poder, gerando muitas das vezes alguns conflitos psicológicos, e sendo um dos gatilhos relevantes para o surgimento dos TCA (Moreira et al., 2017).

Os TCA se caracterizam por graves perturbações no comportamento alimentar. Há uma prevalência crescente de adolescentes, do sexo feminino, impulsionados pelo medo de engordar, motivados pelos paradigmas que a sociedade impõe sobre o padrão de beleza (Pereira, 2020).

Os fatores mais comuns, entre mulheres adulta e adolescentes, que as afetam para desenvolver, segundo Silva, Lima e Santos (2021, p. 9), os TCA são:

"Entre as mulheres, os transtornos alimentares parecem ser mais comuns naquelas de origem caucasiana, quando comparadas com mulheres negras, cujo transtorno alimentar mais comum, quando ocorre, é a BN com uso de laxantes. Devido às intensas transformações físicas e psicológicas na adolescência, é importante identificar a relação existente entre os TA com fatores associados, mostrando a necessidade de intervenções de profissionais especializados nesta população."

Diante do contexto apresentado o objetivo geral do artigo é destacar, através de uma revisão da literatura, a importância do acompanhamento nutricional na recuperação das adolescentes com Bulimia Nervosa. Aponta-se que objetivos específicos são: elencar como os padrões estéticos afetam o modo de vida das adolescentes que sofrem com Transtornos de Comportamento Alimentares (TCA), conceituar a bulimia nervosa e suas classificações; e destacar o papel da boa alimentação e do acompanhamento nutricional em adolescentes com bulimia nervosa.

$\mathrm{O}$ artigo tem como intuito contribuir com conhecimento científico para a comunidade acadêmica, despertando o interesse a reflexão do paciente com BN sobre a semiologia desse transtorno alimentar na adolescência, seus familiares, e profissionais da saúde, quebrando paradigmas já que os pacientes de BN têm uma visão dicotômica e errônea sobre conceitos nutricionais mitos e crenças distorcidas sobre alimentos e alimentação, visando uma melhor percepção sobre o conceito de uma alimentação saudável que ajude melhorar sua qualidade de vida e bem estar. 
A metodologia aplicada para o desenvolvimento desse estudo consiste em uma revisão da literatura fundamentada em artigo, dissertação e teses da área de nutrição. Todos os trabalhos utilizados possuem relação direta com a bulimia nervosa em adolescentes do sexo feminino.

\section{Metodologia}

A pesquisa é uma revisão bibliográfica, do tipo narrativa, sendo este um método de grande relevância no campo da saúde por proporcionar a busca e uma avaliação crítica e a síntese de evidências sobre um tema investigado.

Para a realização deste trabalho, a metodologia adotada foi levantamento bibliográfico que abordassem a temática, através de artigos científicos, obtidos por meio das bases de dados: Scientific Electronic Library Online (SCIELO), Pubmed, Biblioteca Virtual De Saúde (BVS), Google acadêmico entre outros especialistas que abordam esse assunto.

Os descritores usados foram: Bulimia Nervosa, Transtornos Alimentares, Alimentação Saudável, Bulimia Nervosa, Eating Disorders, HealthyEating. Bulimia Nerviosa, Trastornos de la Alimentación, e Alimentación Saludable.

Foram inelegíveis da amostra os artigos publicados e que não estão disponíveis de forma gratuita e não apresentaram o texto na íntegra, artigos que não apresentavam relação direta com o tema, estudos repetidos, além de pesquisas não publicadas entre 2014 e 2021. Os estudos elegíveis foram publicados com menos de 7 anos, em idiomas nacionais e estrangeiros, sendo disponível de forma gratuita e na íntegra e estão relacionados ao objetivo proposto.

Figura 1 - Funil dos estudos selecionados para a pesquisa.

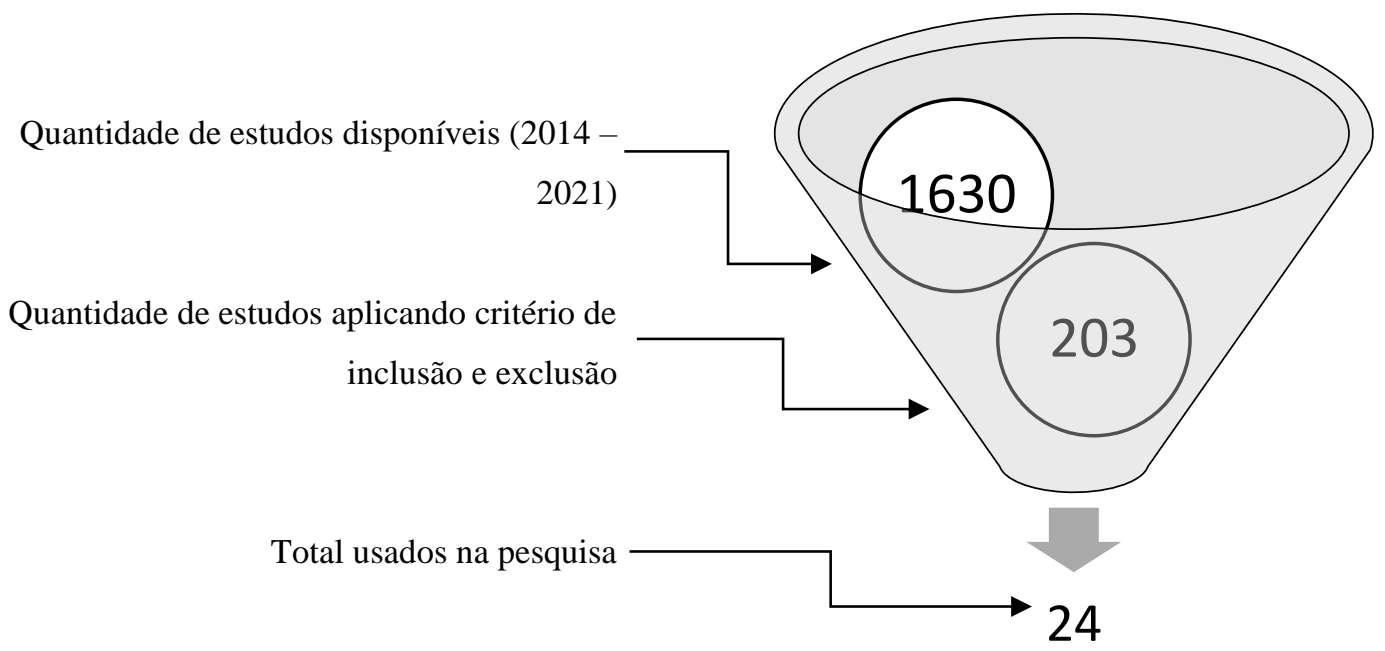

Fonte: Autores (2021).

A Figura 1 evidência que ao todo foram encontrados 1630 estudo que tratavam sobre a bulimia nervosa, após o critério de inclusão e exclusão o esse total $(n=1630)$ foi reduzido para 203. Porém para essa pesquisa, considerando o objetivo do estudo, foram usadas penas 24 estudos. 


\section{Resultados e Discussão}

\subsection{Transtornos de Comportamento Alimentares em adolescentes}

Os TCA tornaram-se nas últimas décadas um problema de Saúde Pública devido ao aumento de novos casos. eu caracterizado por extrema preocupação com peso e aparência física, que resulta em uma deterioração progressiva do organismo devido a uma má absorção de nutrientes dos alimentos (Dias, 2021).

Nunes, Santos e Souza (2017, p. 61) conceituam os TCA como: “[...] Os Transtornos de Comportamento Alimentar (TCA) são denominados como distúrbios psiquiátricos de etiologia multifatorial, caracterizados por consumo, padrões atitudes alimentares e extremamente distorcidas e de preocupação exagerada com o peso e a forma corporal".

As faixas etárias mais suscetíveis aos fatores de risco para transtornos alimentares são adolescentes, já que nesta época da vida o ser humano passa por uma série de modificações em todos os níveis; biológico, psicológica e também na esfera social ao começar a integrar (Nunes, Santos \& Souza, 2017).

Quanto à etiologia dos transtornos alimentares, Silva e Gontijo (2021) referem-se à desregulamentação em fatores neurobiológicos, psicológicos: personalidade, e comportamento. Em relação aos fatores socioambientais, a influência sociocultural ligada à imagem corporal e uma evidente predisposição genética que normalmente acelerado por fatores socioambientais

No que diz respeito à sintomatologia a nível psicológico, existem alterações na percepção da aparência física, pensamentos obsessivos sobre a magreza e na busca pela imagem idealista e o medo de ganho de peso (Barbosa, Lima \& Da Paixão Eneterio, 2018).

Esta sintomatologia carrega, entre outras coisas, à prática de comportamentos purgativos, como o uso indiscriminado de laxantes, à prática excessiva de exercícios físicos, à realização jejuns prolongados e em geral a qualquer conduta que tenha a finalidade de não absorção de alimentos para não ganhar peso corporal (Kunde et al., 2017).

Um estágio avançado de transtorno alimentares, pode causar múltiplas patologias ou problemas, tanto agudos como crônica, de forma progressiva e em vários aparelhos e sistemas do corpo, sendo capaz de chegar à morte (Gómez Candela et al., 2020).

Entre os adolescentes o TCA é desenvolvido, segundo Ludewig et al. (2017) pelos estereótipos sociais, na qual a magreza é valorizada como atratividade físico para alcançar uma forma de sucesso e aceitação social. Imagem corporal feminina e coloca em risco quando as mulheres tentam imitar as características mostradas na publicidade, pois muitos não se importam ou não percebem que colocam em risco sua saúde. Essas mulheres que incorporam essas certas características do modelo magro tendem a positivamente se autoavaliam, enquanto as mulheres que acham inatingível ter o corpo perfeito tem maior probabilidade de cair em anorexia ou bulimia.

\subsection{Conceito de bulimia nervosa}

De acordo com o Manual Diagnóstico e Estatístico para Transtornos Mentais, Quarta Edição (DSM-IV), a BN é caracterizada por episódios recorrentes de compulsão alimentar seguidos de um ou mais comportamentos compensatórios para eliminar as calorias (vômitos, laxantes, jejum etc.) que ocorrem em média um mínimo de duas vezes por semana por três ou mais meses (Nascimento et al. 2014).

Para Chimbinha (2019, p.5) a BN pode ser dita como:

“[...] A bulimia nervosa é mais difícil de ser reconhecida, pois os individuos em geral sentem vergonha de seus problemas alimentares e tentam esconder os sintomas. Nesses casos, a compulsão alimentar normalmente ocorre em segredo ou da maneira mais discreta possível, não apresentam sinais da doença e a maioria está em seu peso normal, caracterizando-se por uma ingestão compulsiva e rápida de grande quantidade de alimentos, com pouco ou nenhum 
prazer, alternando-se com comportamentos dirigidos a evitar o ganho de peso, como por exemplo, vomitar, usar excessivamente laxantes e diuréticos, além de submeter-se a períodos de restrição alimentar severa ou prática de exercício físico intenso".

Adolescentes com BN muitas vezes se envolvem em dietas extremas para neutralizar seus pensamentos desordenados de estar acima do peso. Isso pode levar à compulsão alimentar, que são realizados em segredo e trazem consigo sentimento de culpa e vergonha. Depois de comer uma enorme quantidade de alimentos, as meninas então tentam evitar ganhar peso purgando os alimentos, na maioria das vezes através de vômitos autoinduzidos, às vezes incluindo abuso laxante ou exercícios excessivos (Pereira, 2020).

Um indicador comum de um transtorno de BN em desenvolvimento é uma obsessão por alcançar uma forma corporal e peso "ideais", não importa o quão inatingível possa ser. Perfeccionismo desse tipo leva a um processo de autoavaliação negativa e distorcida que alimenta a continuação de comportamentos desordenados (Bonini et al., 2018).

Existem dois tipos de BN, Meule, Von Rezori e Blechert (2015) conceituam essas classificações da seguinte maneira: Tipo de purgação - O indivíduo sucumbe em comportamentos de abuso medicamentoso, como laxantes, diuréticos ou ele faz enemas, e finalmente, ele se envolve em vômitos autoinduzidos durante a bulimia episódio nervoso. Tipo de não-purgação - O indivíduo adota outros comportamentos compensatórios, como o jejum, exercício físico excessivo durante episódios BN, mas ele não usa laxantes ou envolve vômitos autoinduzidos.

Da mesma forma, a BN pode apresentar uma série de sintomas físicos causados pelo atos compensatórios, entre os quais há evidências problemas do sistema nervoso central (apatia, desregulação emocional), problemas cardiovascular (hipotensão, tontura, desequilíbrios eletrolíticos), nos músculos esquelético (retardo de crescimento), gastrointestinal (erosões na boca, perdas dentes, ruptura do esôfago), sistema endócrino (distúrbio da tireoide, intolerância a frio, baixa temperatura corporal, perda de peso, queda de cabelo e / ou aumento do cabelo corpo) e complicações associadas ao abuso de laxantes (insuficiência renal, cólon catártico, desidratação (Chacho, 2020).

Além disso, BN apresenta características psicológicas como preocupação excessiva por peso e aparência física, labilidade emocional, impotência; falta de controle, estados de humor deprimido, vergonha de ações, ansiedade, irritabilidade, uso de substâncias, pensamentos suicidas, percepção equivocada da aparência física, insatisfação com sua figura do corpo, autocrítica sobre si próprios e tendem a não ter autoestima (Pereira, 2020; De Moraes, De Almeida Maravalhas \& Mourilhe, 2019).

Fazer o diagnóstico de BN não é fácil, pois o paciente tende a praticar secretamente o quadro e pode apresentar peso normal, ou mesmo sobrepeso ou obesidade, sendo mais difícil de detectar, e o primeiro contato com o profissional de saúde é tardio. Muitas vezes, os pacientes buscam médicos especialistas com diferentes quadros somáticos inespecíficos, sendo comum relato de fraqueza, falta de jeito, cansaço, menstruação irregular, dor e distensão abdominal, além de constipação. Diante disso, podem ser diagnosticados de forma errada e, consequentemente, não são tratados de forma correta (Gómez Candela et al., 2020).

\subsection{A importância do acompanhamento nutricional na recuperação das adolescentes com Bulimia Nervosa.}

A atenção correta aos transtornos alimentares, como a BN, requer a intervenção de especialistas de várias disciplinas que trabalham de forma coordenada. Podem participar do tratamento: psiquiatras, psicólogos, médicos de família, nutricionistas, enfermeiras, terapeutas ocupacionais e de imagem e cada um contribuirá com uma parte importante de suas especialidades (De Moraes, De Almeida Maravalhas \& Mourilhe, 2019).

Diferentes profissionais participam das equipes de nutrição para o tratamento da $\mathrm{BN}$, com diferentes treinamentos, responsabilidades e tarefas atribuídas. Em geral participam o médico especialista e o especialista em nutrição clínica. Em 
qualquer caso, o nutricionista deve estar em constante comunicação com as equipes de atenção primária e com os demais especialistas envolvidos no tratamento do paciente (Le Grange \& Lock, 2020).

$\mathrm{O}$ paciente com $\mathrm{BN}$ deve estar sob supervisão e tratamento do nutricionista, que fará o diagnóstico diferencial correto e será responsável por avaliar seu estado nutricional e possíveis complicações somáticas associadas. Também estabelecerá o tratamento nutricional e farmacológico mais adequado, bem como a necessidade ou não de consulta a outros especialistas (Gómez Candela et al., 2020).

Bulimia, em adolescentes, geralmente é tratada com uma combinação de terapia individual, terapia familiar, modificação de comportamento e reabilitação nutricional. (Silva, Lima \& Santos, 2021). O tratamento deve sempre ser baseado em uma avaliação minuciosa do adolescente e da família. A incidência frequente de complicações médicas durante o curso da reabilitação requer que tanto o profissional de saúde quanto o nutricionista sejam membros ativos da equipe de manejo (De Moraes, De Almeida Maravalhas \& Mourilhe, 2019).

O paciente precisará de ajuda para desenvolver uma relação mais saudável com a comida, evitando assim os comportamentos compensatórios. De Moraes, De Almeida Maravalhas e Mourilhe (2019, p. 28) compreendem o tratamento da $\mathrm{BN}$ tem como principal intuito:

"[...] O principal objetivo do tratamento nutricional na BN é auxiliar o paciente a manter um padrão de refeições estruturadas. Dessa forma, é possível reduzir os seguintes comportamentos que caracterizam a BN: 1) restrição alimentar; 2) episódios de compulsão alimentar; 3) comportamentos compensatórios. Nesse contexto, o aconselhamento nutricional desempenha um papel importante no tratamento multiprofissional. As orientações nutricionais auxiliam a redução dos comportamentos alimentares inadequados, da restrição alimentar, dos métodos compensatórios e o aumento da variedade dos alimentos consumidos".

O adolescente ao ser diagnosticada com BN pode apresentar dois quadros clínicos de desnutrição ou de sobrepeso. Nos casos de desnutrição, o nutrólogo será o principal responsável por restaurar o estado mínimo de saúde para que o tratamento psiquiátrico seja viável. Nos casos em que o paciente está em consulta por sobrepeso é possível que direcionado através dos programas de obesidade. De qualquer forma, esses pacientes com BN devem ser encaminhados para que seja feito um diagnóstico e indicado um tratamento altamente individualizado no contexto da complexidade desta patologia (Silveira, 2021).

Segundo Santos $(2021$, p.6) a intervenção nutricional e mulheres adolescentes com BN deve ser orientada da seguinte forma:

“[...] A intervenção nutricional deverá ser gradativa e por etapas, de acordo com as prioridades detectadas por meio de diário alimentar. Introduzem-se, portanto, tarefas objetivas para a mudança do comportamento alimentar e a cessação dos métodos compensatórios, como estabelecer horários para as refeições, não omitir refeições, mastigar bem os alimentos, visualizar as porções servidas das refeições e dos lanches que serão consumidos".

Uma alimentação nutritiva e equilibrada pode melhorar o desempenho do organismo, garantir a saúde, prevenir doenças e aumentar a longevidade. Para isso, a alimentação precisa ser variada, composta por elementos de todos os grupos de macronutrientes, pois cada um deles desempenha funções específicas no organismo. Se toda a família tiver uma alimentação saudável, será muito mais fácil para a adolescente incorporar esses hábitos ao seu dia a dia (Coelho, 2020).

Os nutricionistas indicarão alimentos que contenham as vitaminas e minerais que estão faltando no organismo e cuja ingestão se faça necessária, nas adolescentes mulheres diagnosticadas com BN (De Moraes, De Almeida Maravalhas \& Mourilhe, 2019).

O nutricionista participará da capacitação da família em orientações nutricionais e comportamentos alimentares, bem como no suporte nutricional e aconselhamento sobre atitudes e medidas a serem tomadas em relação à alimentação dos pacientes. E deve estará atento ao estado mental do paciente e garantirá que a atividade terapêutica foi iniciada a esse respeito. Além disso, 
caberá a indicar a incorporação do paciente ao Programa de Educação Nutricional, escolhendo o momento adequado para que essa intervenção seja mais efetiva (Silva, Lima \& Santos, 2021).

A educação nutricional (EN) dos adolescentes com BN deve ser realizada por profissionais qualificados e especialistas e deve fazer parte do tratamento dessa doença. O nutricionista é o profissional mais indicado para realizar a tarefa de BN, mas deve manter a comunicação com o restante da equipe (Farias \& Rosa, 2020).

Outra abordagem usada pelo nutricionais para tratar jovens mulheres com BN é a terapia cognitivo-comportamental (TCC). A TCC é uma abordagem psicoterapêutica que envolve uma variedade de técnicas. Essas abordagens ajudam um indivíduo a compreender a interação entre seus pensamentos, sentimentos e comportamentos e desenvolver estratégias para mudar pensamentos e comportamentos a fim de melhorar o humor e o funcionamento (Moreira et al., 2017).

De acordo com esse modelo cognitivo, o tratamento para bulimia não se concentra apenas nos comportamentos de apresentação de compulsão alimentar e purgação, mas trata dos fatores que mantêm a condição. O tratamento, portanto, usa uma combinação de procedimentos cognitivos e comportamentais para mudar o comportamento dos pacientes e suas cognições disfuncionais sobre peso e forma, enquanto substitui a restrição alimentar por padrões de alimentação mais regulares e saudáveis (Farias \& Rosa, 2020).

Nos casos mais graves, o nutricionista também é responsável por fazer a indicação de internação hospitalar quando a situação clínica for suficientemente grave nos casos mais complicados (comportamentos purgativos descontrolados, complicações médicas graves, autolesões, psicose, etc.), juntamente com a psiquiatria (De Moraes, De Almeida Maravalhas \& Mourilhe, 2019).

\section{Considerações Finais}

A bulimia nervosa é um distúrbio que causa o consumo de quantidades exorbitantes de alimentos com atos de limpeza em um período aproximado de uma vez por semana por três meses, atinge mais a população adolescente, principalmente o gênero feminino, causando uma série de sintomas físicos e psicológicos que interferem na vida diária da pessoa.

Os fatores envolvidos na manutenção do transtorno BN são biológicos como predisposição genética, gênero. Os psicológicos incluem impulsividade, perfeccionismo, baixa autoestima, entre outros. Já o sociocultural, e os sociais são a influência da mídia; e relações e dinâmicas do contexto intrafamiliar. A bulimia nervosa implica no tratamento individualizado, porém acompanhado por uma equipe equipes multiprofissionais.

Várias ferramentas terapêuticas podem ser utilizadas para o tratamento de pacientes com bulimia nervosa que inclui aconselhamento dietético individualizado que garante um estado nutricional adequado. Educação nutricional correta, com o objetivo de objetivo é facilitar a adoção voluntária de comportamentos alimentares que saudáveis e que permite a modificação a longo prazo dos hábitos alimentares e a cessação de comportamentos purgativos e de compulsão alimentar.

Em suma, a nutrição tem um papel crucial, não necessariamente para ensinar a comer o alimento saudável e, sim buscar as possibilidades para que a paciente mude sua atitude em relação ao alimento e a imagem corporal.

Para trabalhos futuros sugere-se pesquisa na qual investiguem outros transtornos alimentares entre os adolescentes, pois como evidenciado as redes sociais vem influenciado como esses públicos se enxergam, que por sua vez, afetam a forma como se alimentam na busca pelo corpo considerado ideal na internet.

\section{Agradecimentos}

Agradecemos a todos que direta ou indiretamente contribuíram para a realização e sucesso do artigo. 
Research, Society and Development, v. 10, n. 15, e62101522576, 2021

(CC BY 4.0) | ISSN 2525-3409 | DOI: http://dx.doi.org/10.33448/rsd-v10i15.22576

\section{Referências}

Barbosa, A., Lima, H., \& da Paixão Eneterio, N. G. (2018). A atuação do psicólogo em paciente com anorexia e bulimia nervosas. CIPEEX, 2 , $1962-1968$.

Bonini, A. F., Alckmin-Carvalho, F., El Rafihi-Ferreira, R., \& da Silva Melo, M. H. (2018). Evolução dos critérios para o diagnóstico de Bulimia Nervosa: revisão sistemática. Revista Brasileira de Psicoterapia, 20(2), 65-83.

Chimbinha, I. G. M. et al (2019). Transtornos alimentares e manifestações orais em adolescentes. Revista Ciência Plural, 5(3), 1-20.

Chacho, D. C. (2021). Processo diagnóstico e avaliação do transtorno bulimia nervosa na adolescência, análise de um caso clínico. Trabalho de Conclusão (Psicologia) - Faculdade de Ciencias sociais.

Coelho, J. D. S. C. D. V. (2020). Educação alimentar no ensino médio: abordagens alternativas com foco na alimentação saudável. Trabalho Acadêmico de Conclusão de Curso (Ciências Biológicas) - Universidade Federal da Paraíba.

de Moraes, C. E. F., de Almeida Maravalhas, R., \& Mourilhe, C. (2019). O papel do nutricionista na avaliação e tratamento dos transtornos alimentares. Debates em Psiquiatria, 9(3), 24-30.

Dias, A. S. T. (2021). Tratamento da Perturbação de Ingestão Alimentar Compulsiva em crianças e adolescentes. Dissertação (Mestre em Medicina) Universidade do Porto, 2021.

Farias, C. T. S., \& Rosa, R. H. (2020). A educação alimentar e nutricional como estratégia no tratamento dos transtornos alimentares. Brazilian Journal of Health Review, 3(4), 10611-10620.

Ferreira, I. M. S. Comportamentos de risco nutricional de mães e sua relação com o desenvolvimento do transtorno alimentar de suas filhas (Doctoral dissertation, Universidade de São Paulo).

Gómez Candela, C., Palma Milla, S., Miján de la Torre, A., Rodríguez Ortega, P., Matía Martín, P., Loria Kohen, V., \& Martín Palmero, Á. (2018). Consenso sobre la evaluación y el tratamiento nutricional de los trastornos de la conducta alimentaria: bulimia nerviosa, trastorno por atracón y otros. Nutrición Hospitalaria, 35(SPE1), 49-97.

Nascimento, M. I. C., Machado, P. H., Garcez, R. M., Pizzato, R., Rosa, S. M. M. D., American Psychiatric Association, \& American Psychiatric Association. (2014). Manual diagnóstico e estatístico de transtornos mentais: DSM-5. Associação Brasileira de Psiquiatria. Artmed.

Kunde, F. R., Mitinguel, L. H., Bellato, A., \& Moreira, M. A. (2017). Perimólise em paciente portadores de bulimia nervosa do tipo purgativa: Revisão de Literatura. Conversas Interdisciplinares, 13(3).

Le Grange, D., \& Lock, J. (2020). Tratando bulimia em adolescentes: Uma abordagem baseada no envolvimento de toda a família. M. Books do Brasil Editora,

Ludewig, A. M., Rech, R. R., Halpern, R., Zanol, F., \& Frata, B. (2017). Prevalência de sintomas para transtornos alimentares em escolares de 11 a 15 anos da rede municipal de ensino da cidade de Nova Petrópolis, RS. Revista da AMRIGS, 61(1), 35-39.

Meule, A., von Rezori, V., \& Blechert, J. (2014). Food addiction and bulimia nervosa. European Eating Disorders Review, 22(5), $331-337$.

Moreira, G. S. X., Boff, R. M., Pessa, R. P., Oliveira, M. S., \& Neufeld, C. B. (2017). Alimentação e imagem corporal. Terapia Cognitivo-Comportamental para adolescentes: uma perspectiva transdiagnóstica e desenvolvimental, 150-187.

Nunes, L. G., Santos, M. C. S., \& Souza, A. A. (2017). Fatores de risco associados ao desenvolvimento de bulimia e anorexia nervosa em estudantes universitários: uma revisão integrativa. HU Revista, 43(1).

Pereira, S. I. S. (2018). Uma viagem à etiologia multifatorial da anorexia nervosa: determinantes biológicos, psicológicos, genéticos e socioculturais. Revista Brasileira Interdisciplinar de Saúde, 2020.

Santos, N. F. H. (2021). Bulimia em adolescentes praticantes de atividade física. Research, Society and Development, $10(6)$, e29110615874-e29110615874.

Sebastião, J. R., Sampaio, D., \& Barbosa, M. R. (2018). Prevalência e fatores de risco das Perturbações do Comportamento Alimentar, em adolescentes dos 12 aos 18 anos, no Concelho de Manteigas. Psilogos, 16(2), 31-45.

Silveira, I. C. D. (2021). Abordagens da nutrição comportamental em mulheres com anorexia e bulimia nervosa: revisão narrativa. Abordagens da nutrição comportamental em mulheres com anorexia e bulimia nervosa: revisão narrativa. Monografia (curso de nutrição) - Faculdade Maria Milza,

de Santana Silva, M., \& Gontijo, D. T (2021). Alterações ocupacionais relacionadas à vivência da anorexia e bulimia nervosa por adolescentes: Revisão de escopo. Research, Society and Development, 10 (6), e30310615835-e30310615835.

Silva, A. B., Lima, K. J. S., \& Santos, R. H. (2021). A contribuição da nutrição para o tratamento da bulimia nervosa: revisão integrativa. Trabalho de Conclusão de Curso (Nutrição) - Centro Universitário Tiradentes - UNIT/AL. 